\section{The nitty without the gritty}

\author{
Analysis of Genes and Genomes \\ RJ Reece \\ John Wiley and Sons Ltd, West Sussex, UK; 2003. 469 pp. \\ $£ 34.95$, paperback. ISBN 0470843802.
}

Heredity (2005) 94, 383. doi:10.1038/sj.hdy.6800626

\section{Reviewed by S Hardy}

This is a textbook that covers the nitty of Molecular Biology - the rationale and methods of most recombinant DNA manipulations - without much of the gritty - the experimental protocols. In my entire life, I have read with close attention only three textbooks from cover to cover, one when I was a graduate student - Gunther Stent's brilliant 'The Molecular Biology of Bacterial Viruses' - and two others, including this one, for the purpose of review. A common, and for me surprising, feature of the latter two is that in spite of being good books overall, they are both spattered with errors, mostly minor ones. Presumably this is true of the majority of textbooks, a point that should be emphasised to all students.

Normally I, and probably most other professional scientists, use textbooks as a source of information on topics that are outside our immediate field of expertise. Thus a good textbook must have an excellent index, must contain a comprehensive coverage of the overall topic it purports to address, must be accurate and clearly written, and as a bonus should stimulate interest. On most of these criteria, Richard Reece does well. Many topics that I only vaguely understood are now clear to me. Almost everything I looked for in the index was there, and happily his enthusiasm permeates the pages to hold the reader's interest. Furthermore, the list of relevant Nobel laureates at the end will be useful and was a delight.

For undergraduates, who have no established field of expertise, and who are probably slightly or more than slightly confused about most things, there is another important requirement. There should be progressive development through the book from the known to the unknown, from the familiar to the mysterious. This also, Reece does quite well, though the audience he is writing for is never identified. Judging by the students I teach, the book would be an excellent text for a second year biology or biochemistry module on recombinant DNA. For such an audience, the first and by far the longest chapter 'DNA: Structure and function', which is basically the central dogma of Molecular Biology explained in detail, would provide the familiar. I liked the historical approach in this chapter. However, it attempts to cover too much. A leaner fitter chapter would have emerged by omitting $\mathrm{A}$ and $\mathrm{Z}$ form DNAs, linking numbers, the chemical explanation of the hyperchromic effect, the putative mechanism of homologous recombination, the mechanism of RNA splicing, and the mechanism of translation. None of these are important for the rest of the book. In addition, there are some significant errors in this chapter, particularly but by no means exclusively, in the short and weak section on translation. An odd feature is that only in this chapter are the amino acids referred to by their three letter designations, thereafter by the single letter code, which is never otherwise mentioned. This code should have been included in Appendix A1.1 with the amino-acid structures and referred to on its first use.

With the scene now rather too fully set, there follow three excellent chapters on basic tools and techniques. The one on vectors was particularly good with the best description and figure of blue-white screening I have seen, and it was also good that PCR with its splendid versatility got the full chapter it deserved. The next four chapters, which consider libraries, screening, mutagenesis, and protein isolation are the core of the 'Analysis of Genes' section. Highlights for me were the very clear sections on subtraction libraries and on two-hybrid screening. A really excellent feature of Reece's approach that is used here is that on many occasions, having described a system or procedure, he will step back and discuss the disadvantages and problems associated with it. This enhances the interest in, and deepens the understanding of, the feature he is writing about. Once again there is a slight tendency in these seven chapters to introduce unnecessary topics, for example, bacterial conjugation and antibody structure. Being a pedant, I was also irritated by the frequent references to PCR reactions.

The last five chapters cover genome elucidation and analysis followed by engineering of plants and animals, finishing with Dolly and human gene therapy. I enjoyed them, being particularly pleased by the clear description of radiation hybrid mapping, a topic that is given very short shrift or omitted by other comparable texts. On the other hand, I was confused by the examples chosen to illustrate microarray usage and towards the end of the book the frequency of typos seemed to increase. Overall, I detected more than 70 !

This niche is a crowded and competitive one for texts. At $£ 34.95$, the book is between $£ 4$ and $£ 8$ more expensive than the three competitors I looked at, but it has a good supporting website, is more recent, and has multicolour figures. I do not know whether students care about colours but they do care about price. Nevertheless, if I were teaching a module on recombinant DNA this year I would recommend this book because even when finding fault with it I was never bored.

S Hardy

Department of Biology, University of York, PO Box 373, York YO10 5YW, UK

E-mail: sjsh1@york.ac.uk 